\title{
ELABORACIÓN Y ACEPTABILIDAD DE BARRITAS ENERGÉTICO-PROTEICO MULTICEREAL PARA LA LONCHERA ESCOLAR
}

\author{
ELABORATION AND ACCEPTABILITY OF MULTICEREAL \\ ENERGETIC-PROTEIN BARRIERS FOR SCHOOL LAUNCHER
}

Carmen Lali Aponte Guevara1', Guillermo Alejandro Sánchez Guzmán', Norma Elvira Muguruza Crispin', Domingo Chiroque Sernaqué', Rubén Guerrero Romero', Humberto Carreño Mundo',

Ricardo Ramiro Palomino Pezzutti ${ }^{1}$

Se elaboró tres barritas energético-proteico multicereal con harina de trigo, kiwicha, chía, cáscaras de frutas y verduras, para la lonchera escolar.Se utilizò un diseño cuasi experimental, análisis físico, químico según métodos de la AOAC, la aceptabilidad según análisis de varianzas de un solo factor y prueba de comparaciones múltiples de tukey II. en los resultados no existen diferencias significativas en la aceptabilidad global de las barritas formuladas: "multibar-1","multibar-2", "multibar-3". Las barritas energético-proteico multicereal, presentaron un contenido de proteínas, de 9,65 g\% $\pm 0,257$ (Multibar-1), 11,42 g\% 0,326 (Multibar-2) y 11,36 \pm 0,369 (Multibar-3); grasas, de 9,18 g\% $\pm 0,178$ (Multibar-1), 10,83 g\% $\pm 0,874$ (Multibar-2) y 11,14 $\pm 0,326$ (Multibar-3), y fibra alimentaria de 7,58 g\% $\pm 0,215 ; 7,93 \mathrm{~g} \% \pm 0,262$ y $8,16 \mathrm{~g} \% \pm 0,226$, respectivamente. Se Ilego a la conclusion que las barrritas energético-proteico multicereal ("multibar-1", "multibar-2", "multibar-3"), tienen buena aceptabilidad global y buen valor nutritivo. La ingesta de $100 \mathrm{~g}$ de este producto que equivale a aproximadamente 2 barras, cubre el $30 \%$ y $25 \%$ de los requerimientos de proteínas y fibra alimentaria del preescolar y escolar.

Palabras clave: Barritas; Multicereal; Energético-proteico; Aceptabilidad.

\begin{abstract}
Three energy-protein multicereal bars were prepared with wheat flour, kiwicha, chia, fruit and vegetable peels, for the school lunch box. We used a quasi-experimental design, physical analysis, chemical analysis according to AOAC methods, acceptability according to a one-factor variance analysis and multiple comparison test of Tukey II. in the results there are no significant differences in the overall acceptability of the bars formulated: "multibar-1", "multibar-2", "multibar-3". The energy-protein multicereal bars presented a protein content of $9.65 \mathrm{~g} \% \pm 0.257$ (Multibar-1), $11.42 \mathrm{~g} \% \pm 0.326$ (Multibar-2) and $11.36 \pm 0.369$ (Multibar-3) ; fats, of $9.18 \mathrm{~g} \% \pm 0.178$ (Multibar-1), $10.83 \mathrm{~g} \% \pm 0.874$ (Multibar-2) and $11.14 \pm 0.326$ (Multibar-3), and dietary fiber of $7.58 \mathrm{~g} \% \pm 0.215 ; 7.93 \mathrm{~g} \% \pm$ 0.262 and $8.16 \mathrm{~g} \% \pm 0.226$, respectively. It was concluded that the multi-energy energetic-protein bars ("multibar1", "multibar-2", "multibar-3"), have good overall acceptability and good nutritional value. The intake of $100 \mathrm{~g}$ of this product, which is equivalent to approximately 2 bars, covers $30 \%$ and $25 \%$ of the protein and fiber requirements of pre-school and school children.
\end{abstract}

Keywords: Bars; Multicereal; Energetic-protein; Acceptability.

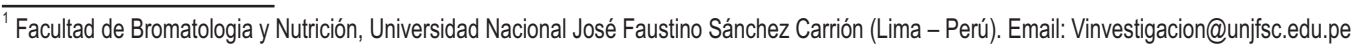




\section{INTRODUCCIÓN}

En el Perú más de la mitad de la población infantil sufre de mal nutrición por diversas razones. Las familias pobres no tienen capacidad adquisitiva para una balanceada alimentación de sus niños. La carne, la leche y otros alimentos de origen animal, son caros, y por eso es importante buscar fuentes proteicas más económicas y hacerlas llegar a la población más necesitada, de allí la importancia de elaborar nuevos productos alimenticios (mezclas instantáneas), sobre la base de cultivos andinos, cuya calidad nutricional (proteico calórica) se acerque a la de origen animal; utilizando el proceso tecnológico de cocción-extrusión, que permite obtener productos de muy buena calidad y aceptabilidad, así como mínima pérdida de componentes nutricionales. Uno de los principales problemas de salud infantil en nuestro país, es la desnutrición crónica existente, originada por la ingesta de una dieta inadecuada (deficiente en proteínas, yodo, hierro y micronutrientes)o por la existencia de una enfermedad recurrente, o la presencia de ambas.

Actualmente, se están desarrollando a nivel industrial y en centros de investigación toda una generación de alimentos saludables, como es el caso de la presente investigación que tuvo como objetivo principal.Determinar los porcentajes adecuados de harina de trigo, kiwicha, quinua, tarwi, y semillas de linaza, chia, girasol, ajonjolí y cáscara de frutas y obtener una barrita, fuente de proteínas y fibra dietaria como nueva alternativa de consumo, dentro del mercado de barras energéticas, que sean del agrado de los niños en la lonchera escolar .

La importancia del presente trabajo de investigación radica en que los resultados que se obtengan permitirá: Incentivar en el poblador peruano el consumo de alimentos de origen andino. Dar valor agregado a productos agrícolas andinos, impulsando el desarrollo del sector agroindustrial.Disminuir la dependencia de alimentos importados (trigo, maíz, etc.) con el consiguiente ahorro de divisas. Proporcionar alternativas de investigación tecnológica agroalimentaria que permita diseñar harinas instantáneas en base a mezcla de recursos autóctonos nutritivos y saludables.

\section{MATERIALES Y METODOS}

Área de estudio:

Tecnología de los Alimentos. Producción de alimentos diseñados.

Localización geográfica:

Distrito de Huacho, Provincia de Huaura. Región LimaProvincias.

Tipo de Investigación:

Descriptivo analítico, de corte transversal y prospectivo.

Nivel de Investigación:

Aplicada.

Materiales y equipos

Población:

Materia prima e ingredientes para la elaboración de las barritas de harina de trigo, kiwicha, semillas de chia y cáscara de frutas y verduras,.
Muestra: Se utilizó cantidades representativas de los ingredientes necesarios para la elaboración de las barritas.

\section{Diseño metodológico.}

Métodos.

Descriptivo: Pruebas sensoriales mediante encuestas y entrevistas personales.

Analítico: Relación de la variable aceptabilidad y, aporte nutricional después del acopio de datos.

- Síntesis: Conclusiones de la investigación.

- Estadística: Descriptiva e inferencial

Elaboración de barritas energético-proteico multicereal para la lonchera escolar.

Se prepararon barritas de harina de trigo, kiwicha, semillas de chia y, cascara de frutas y verduras, con tecnología artesanal adaptado a los requisitos según norma de Codex para alimentos elaborados a base de cereales para lactantes y niños pequeños (Codex Stan 074-1981). NTP. 206.001(1981). Galletas- Requisitos y NTP 205.044 (1976). Harinas sucedáneos procedentes de leguminosas de grano alimenticio.

Recepcionado.

La toma de la muestra, se realizó según el método aleatorio simple.

\section{Seleccionado y pesado}

Se evaluarón las características físico organolépticas de acuerdo a las normas de calidad. Se determinó el peso para evaluar las pérdidas por procesamiento.

\section{Desinfectado y lavado}

Se realizó una operación de desinfección de la materia prima básica para eliminar gran parte de los contaminantes físicos y lavados sucesivos (eliminar la posible presencia de saponinas en la kiwicha, y semillas de chia). Se utilizó solución clorada 20 ppm. y lavado por arrastre.

\section{Acondicionado.}

La kiwicha,y las semillas de chia fueron la principal fuente proteica y de ácidos grasos omegas del producto, por lo tanto el tratamiento estuvo enfocado en la conservación de las características nutricionales, considerando las limitaciones en la textura que podría presentar el producto final.

\section{Formulado}

Se prepararon las barritas energético-proteico multicereal, según nivel de mezcla de harina de trigo, harina pretostada de kiwicha y semillas de chia. Se agregaron cáscaras de frutas (naranja, manzana, mango), y verduras (zanahoria), almendra seca picada y trozos de pasas adquiridos en el comercio. El porcentaje de estos ingredientes correspondió entre 3 a $5 \%$ del total de la formulación para cada uno, completando un $6 \%$ entre la almendra picada y la pasa. En la mezcla aglutinante se agregó proteína de soya (5\%), saborizantes de vainilla y caramelo. Las proporciones utilizadas fueron de $0,60 \%$ para el sabor de vainilla y de $0,60 \%$ para el sabor de caramelo respecto al peso total 
Hipótesis alterna

Ha1= Uno de los productos evaluados es preferido sobre los demás.

$\mathrm{Ha} 2=$ Uno de los productos evaluados tiene mejor valor nutritivo que los demás.

Interpretación:

\section{$\mathrm{Fc}<\mathrm{Ft}$ Se acepta Ho \\ $\mathrm{Fc}>\mathrm{Ft}$ Se rechaza Ho \\ Se acepta $\mathrm{Ha}$}

Análisis sensorial.

Por tratarse de un estudio cualitativo, no se utilizó una muestra probabilística. Los resultados obtenidos no pueden generalizarse a toda la población infantil sino que sirvieron para describir, comparar y caracterizar a las barritas energético-proteico multicereal para su utilización en la lonchera escolar como alternativa a las galletas y similares, tomando como indicador un segmento de la población.

Metodología del grupo focal.

Se planteó el objetivo del grupo focal que fue apreciar las reacciones y sentimientos de los niños acerca de las tres formulaciones de barritas. Luego se diseñaron los criterios de segmentación tomando en cuenta que cada grupo contara con 20 niños escolares (7-12 años de edad). Los niños que se eligieron para este estudio fueron de estatus socioeconómico baja. Luego de tener la lista de los niños a participar se les hizo entrega a cada padre de familia una carta de autorización para que su hijo fuera parte del estudio.

\section{RESULTADOS}

Elaboración y aceptabilidad de barritas energéticoproteico multicereal para la lonchera escolar.

En la figura 1, se muestra los valores promedios de las calificaciones de la aceptabilidad global de las barritas energético-proteico multicereal elaboradas (Multibar-1, multibar-2, multibar-3).

Figura 1: Aceptabilidad global de productos formulados.

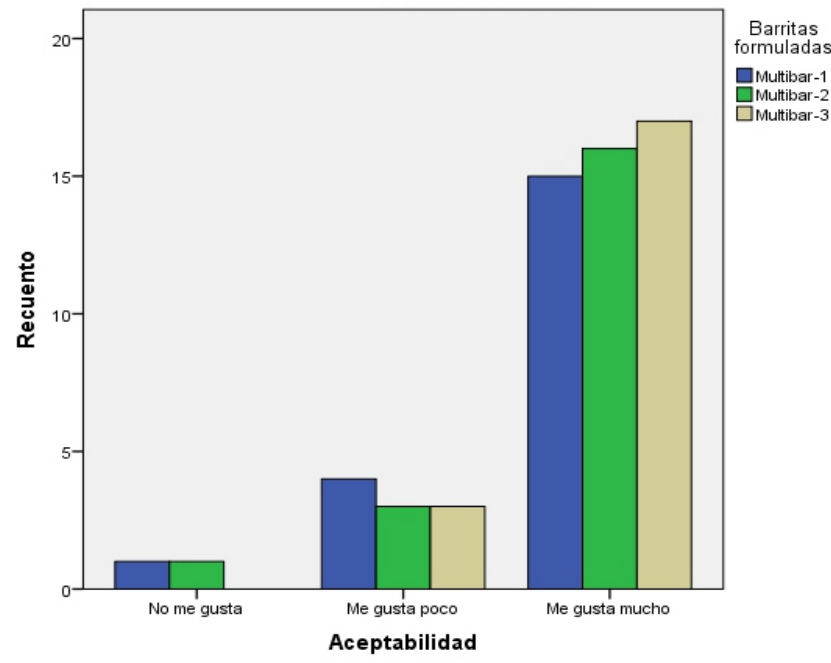

Big Bang Faustiniano 2017; 6(4): Pag. 36 - 41
Tabla 1: ANOVA de barritas energético-proteico multicereal formuladas

\begin{tabular}{|c|c|c|c|c|c|}
\hline & $\begin{array}{l}\text { Suma de } \\
\text { cuadrados }\end{array}$ & $g$ & Media cuadrática & $\mathrm{F}$ & Sig. \\
\hline Entre grupos & 0,233 & 2 & 0,117 & 0,459 & 0,634 \\
\hline Dentro de grupos & 14,500 & 57 & 0,254 & & \\
\hline Total & 14,733 & 59 & & & \\
\hline
\end{tabular}

INTERPRETACIÓN: $\mathrm{p}_{0,95}=0,05$

$\mathrm{Ho}=$ No existen diferencias significativas entre los promedios de la calificación nominal de aceptabilidad de las barritas formuladas $\left(p_{0,95}>0,05\right)$.

u "multibar-1" = u "multibar-2" = u "multibar-3"

$\mathrm{Ha}=\mathrm{Si}$ existen diferencias significativas entre los promedios de la calificación nominal de aceptabilidad de las barritas formuladas $\left(p_{0,95}</=0,05\right)$

u "multibar-1" = u "multibar-2" \# u "multibar-3"

Los valores "p" del estadístico de Fisher experimental $(\mathrm{Fc})$, muestran que el error para aceptar que las tres barritas formuladas, tienen diferente aceptación es demasiado grande $(63,4 \%)$. Es decir que los cambios sensoriales, estadísticamente son no significativos, por encontrarse la probabilidad dentro del área de aceptación del $5 \%$.

Análisis químico proximal comparativo de las barritas energético-proteico multicereal.

La tabla 2, muestra los resultados promedios del análisis químico proximal comparativo realizados en las tres barritas formuladas.

Tabla 2: Composición química proximal de las barritas energético-proteico multicereal.

\begin{tabular}{lccc}
\hline Componentes & \multicolumn{3}{c}{ Barras ( g/100g) } \\
& Multibar-1 & Multibar-2 & Multibar-3 \\
& X \pm DS & X \pm DS & X \pm DS \\
\hline Humedad & $9,83 \pm 0,214$ & $10,12 \pm 0,174$ & $10,58 \pm 0,258$ \\
Proteína & $9,65 \pm 0,257$ & $11,42 \pm 0,326$ & $11,36 \pm 0,369$ \\
Grasas & $9,18 \pm 0,178$ & $10,83 \pm 0,874$ & $11,14 \pm 0,326$ \\
Cenizas & $2,16 \pm 0,021$ & $2,62 \pm 0,023$ & $2,71 \pm 0,019$ \\
Carbohidratos ${ }^{1}$ & $69,18 \pm 1,641$ & $65,01 \pm 1,524$ & $64,21 \pm 1,486$ \\
Fibra dietaria & $7,58 \pm 0,215$ & $7,93 \pm 0,262$ & $8,16 \pm 0,226$ \\
Energía (Kcal \%) & 401,41 & 402,67 & 399,42 \\
pH & 4,5 & 4,5 & 4,5 \\
\hline
\end{tabular}

Las barritas energético-proteico comparado con las barras comerciales, presentaron un mayor contenido de proteínas, de 9,65 g\% $\pm 0,214$ (Multibar-19), 11,42 $\mathrm{g} \% \pm 0,326$ (Multibar-2) y 11,14 $\pm 0,326$ (Multiabr-3), y menor contenido de grasas, de $9,18 \mathrm{~g} \% \pm 0,178$ (Multibar-1), 10,83g\% \pm 0,874 (Multibar-2) y $11,36 \pm$ 0,369 (Multibar-3), en relación al cereal mix $(5,71 \%$ de proteínas y $20,95 \%$ de grasas); barra de sesamo y chia $(9,52 \%$ de proteínas y $14,29 \%$ de grasa), cereal bar (3,75\% de proteínas y $15 \%$ de grasas), y con una variación significativa en el contenido de fibra dietaria a los indicados en las etiquetas nutricionales de cereal 
de la barra, de acuerdo a recomendaciones bibliográficas. En la Tabla 1, se muestra las formulaciones de las pruebas experimentales:

Tabla 1: Formulación base de barritas energéticoproteico multicereal.

\begin{tabular}{|c|c|c|c|}
\hline Ingredientes (\%) & Multibar-1 & Multibar-2 & Multibar-3 \\
\hline Harina de trigo & 20,00 & 25,00 & 20,00 \\
\hline $\begin{array}{l}\text { Kiwicha } \\
\text { (pretostada) }\end{array}$ & 24,00 & 22,00 & 20,00 \\
\hline Chia (semillas) & 7,00 & 7,00 & 10,00 \\
\hline Naranja (cáscara) & 4,00 & 4,00 & 4,00 \\
\hline $\begin{array}{l}\text { Manzana } \\
\text { (cáscara) }\end{array}$ & 4,00 & 3,00 & 5,00 \\
\hline Mango (cáscara) & 4,00 & 3,00 & 5,00 \\
\hline $\begin{array}{l}\text { Zanahoria } \\
\text { (cáscara) }\end{array}$ & 3,00 & 3,00 & 5,00 \\
\hline Almendras & 3,00 & 3,00 & 3,00 \\
\hline Pasas & 3,00 & 3,00 & 3,00 \\
\hline Proteína de soya & 5,00 & 5,00 & 5,00 \\
\hline Leche en polvo & 4,00 & 4,00 & 4,00 \\
\hline Aceite vegetal & 5,00 & 5,00 & 5,00 \\
\hline Miel de abeja & 12,00 & 12,00 & 12,00 \\
\hline Esencia vainilla & 0,60 & 0,60 & 0,60 \\
\hline Caramelo & 0,60 & 0,60 & 0,60 \\
\hline Sal & 0,70 & 0,70 & 0,70 \\
\hline $\begin{array}{l}\text { Benzoato } \\
\text { sodio }\end{array}$ & 0,10 & 0,10 & 0,10 \\
\hline Agua & c.s.p. & c.s.p. & c.s.p. \\
\hline
\end{tabular}

Fuente: (c.s.p.) Agua en cantidad necesaria para formación de la masa

Se tomó como referencia la variable sensorial: Sabor. La concentración final de sólidos totales fue de $90 \%$, como mínimo. La adición de ácido ascórbico $(500 \mathrm{mg} / \mathrm{Kg}$ de masa) y benzoato de sodio $(0,10 \%)$ se utilizó para inhibir el crecimiento de mohos y levaduras.

Mezclado y homogenizado

La kiwicha tostada, semilla de chia y cáscara de frutas (naranja, manzana y mango) y verduras (zanahoria), confitadas y seca, se mezclaron, para luego ser adicionadas a la masa laminada.

Laminado y cortado

El producto fue laminado con un rodillo de madera y luego cortado en formato rectangular de $5 \mathrm{~cm} \times 2 \mathrm{~cm} \times$ $0,5 \mathrm{~cm}$.

Horneado

A $160^{\circ} \mathrm{C}$, por un tiempo de 15 minutos hasta alcanzar una humedad final no mayor del $10 \%$.

Envasado y sellado

Se llevó a cabo manualmente utilizando bolsas de polietileno revestidas con papel aluminio.

\section{Etiquetado}

En los envases se colocaron las respectivas etiquetas donde se mencionan los ingredientes utilizados en la preparación, su composición química, propiedades naturales, fecha de elaboración y tiempo límite que el producto podrá ser consumido.

\section{Almacenado}

El producto fue almacenado en ambientes adecuados, a temperatura ambiente, durante 30 días. Los resultados fueron utilizados para el análisis estadístico.

Control de Calidad

--Caracteres organolépticos:

Método sensorial. AOAC.

-Análisis Físico

Determinación de humedad:MétodoAOAC.

Determinación del pH:MétodoAOAC.

-Análisis Químico Bromatológico

Determinación de acidez titulable: Método AOAC

determinación de proteínas totales:método Kjeldahl. AOAC

Determinación de extracto étereo:

Método Soxhlet.AOAC determinación de carbohidratos:

Método Nifext. AOAC determinación de fibra alimentaria:

Método Químico - Enzimático. AOAC

Determinación de cenizas: métodoAOAC.

Determinación del valor calórico:método Atwater.

-Análisis microbiológico.

Recuento de aerobios mesófilos viables:Método Norteamericano SPC (ICMSF 2006).

Recuento de mohos:

Método Howard (ICMSF 2006).

Recuento de coliformes:

Método Norteamericano-. Número más probable (ICMSF 2006).

Análisis estadístico para la contrastación de hipótesis.

Se formularon:

Hipótesis general:

$\mathrm{H} 1$ : Las barritas energético proteico multicereal elaboradas con tecnología artesanal son del agrado de los niños, y pueden sustituir a las galletas en la lonchera escolar por su valor nutricional.

Hipótesis Secundaria:

H1: Las barritas energético - proteico multicereal, tienen buen valor nutritivo para ser utilizado en la lonchera escolar.

Hipótesis nula

Ho1= No existe diferencias significativas entre los tres productos elaborados (Multibar-1, Multibar-2, Multibar$3)$.

$\mathrm{Ho} 2=$ No existe diferencias significativas entre el valor nutritivo de los tres productos formulados escolar (Multibar-1, Multibar-2, Multibar-3), para ser utilizado en la lonchera 
mix (Arcor), cereal bar (Costa) y barritas de sesamochia (Cereales andinolas barritas con fines comerciales, muchas de ellas s).

La industrialización con grasas trans, colorantes, saborizantes y azúcares, presentan productos de buen sabor pero de bajo valor nutritivo, que son consumidos masivamente por los escolares.Larrabure. (2008), reporta que entre el $7 \%$ y $12 \%$ de niños menores de 05 años y hasta un $28 \%$ en edad escolar presentan exceso de peso u obesidad, debido al elevado consumo de azúcares simples y de grasa (en forma de galletas y pastelillos), lo que ocasiona un aporte elevado de energía en la dieta. Reportes de la Asociación de Consumidores de Lima-Metropolitana (2011) muestran que los bocaditos (palitos de maíz, papas fritas, maíz frito y camote frito), pastelillos (golpe, chocman, kekitos), significan para los niños un exceso en promedio del $30 \%$, equivalente a aproximadamente $700 \mathrm{Kcal} / \mathrm{día}$, que son la causa principal de la obesidad que presentan. La mayoría de las familias de esta región incluyen las galletas dentro de los primeros diez productos de consumo en la dieta habitual, como acompañamiento en las principales comidas, sustituyendo al pan (INE, 2010).

Al comparar los resultados de la composición química de las barritas energético-proteico multicereal, lo ubica frente a las barras comerciales, en un rango mayor, el producto se podría comercializar en calidad de snack para niños o para incluirlo en las meriendas escolares aprovechando el gusto de los infantes por productos dulces, lo que brinda al producto elaborado características competitivas para el segmento de mercado que consume alimentos más saludables y bajos en grasa saturadas.

Trabajos recientes muestran que en la mayoría de los productos comerciales el promedio del contenido de proteínas es de tan sólo $5,5 \%$, y su calidad proteica sería pobre en la medida que provienen principalmente de cereales (arroz, avena, maíz). Las grasas utilizadas se encuentran lejos de las recomendaciones actuales, con ausencia de ácidos grasos poliinsaturados y frecuente presencia de grasas saturadas y/o aceites vegetales hidrogenados (Rev. Consumer Internacional, 2008). Éstos últimos pueden aportar en algunos casos, ácidos grasos trans cuyos niveles en una sola unidad de barras comerciales pueden cubrir un elevado porcentaje del máximo admisible establecido por OMS para la dieta: $1 \%$ de la energía total (FAO, 2008). En consecuencia, a pesar del posicionamiento como alimentos saludables, su gran difusión y vertiginoso aumento de la producción a nivel mundial, las barras comerciales están lejos de responder a la calidad nutricional que potencialmente podrían presentar (Asociación de Consumidores y Usuarios de Chile, 2010).

Análisis microbiológico de las barritas energéticoproteico multicereal.

La tabla 3, muestra los resultados promedios del análisis microbiológico (03 repeticiones), realizados en las barritas energético-proteico multicereal formuladas.
Tabla 3: Análisis microbiológico de barritas energéticoproteico multicereal.

\begin{tabular}{|c|c|c|c|c|c|c|}
\hline \multirow[t]{2}{*}{ REFERENCIA } & \multicolumn{3}{|c|}{5 días } & \multicolumn{3}{|c|}{30 días } \\
\hline & Chiak $_{1}$ & Chiak $_{2}$ & $\mathrm{Chiak}_{3}$ & Chiak $_{1}$ & Chiak $_{2}$ & $\mathrm{Chiak}_{3}$ \\
\hline $\begin{array}{l}\text { Numeración de Aerobios } \\
\text { Mesófilos Viables (UFC/g.) V }{ }^{0} N^{0}= \\
10^{4} \cdot 10^{5 *}\end{array}$ & $<10$ & $<10$ & $<10$ & $10^{2}$ & $10^{2}$ & $10^{2}$ \\
\hline $\begin{array}{l}\text { Numeración de Hongos (UFC/g) } \\
V^{0} N^{0}=<10^{3 *}\end{array}$ & $<10$ & $<10$ & $<10$ & $<10$ & $<10$ & $<10$ \\
\hline $\begin{array}{l}\text { Numeración Co liformes (NMP/g) } \\
V^{0} N^{\circ}=<3^{*}\end{array}$ & 0 & 0 & 0 & 0 & 0 & 0 \\
\hline
\end{tabular}

UFC= Unidad formadora de colonia; NMP= Número más Probable (1) Especificaciones Técnicas: Norma Técnica Peruana 031 (2)* Según Codex Alimentarius (3) Norma sanitaria de Criterios Microbiológicos de Calidad Sanitaria e Inocuidad para los alimentos y bebidas de consumo humano. (DIGESA -Ministerio de Salud. Lima Perú. 2008)

Los resultados microbiológicos, representados por el recuento total de anaerobios mesófilos, hongos y numeración de coliformes, indican que el producto elaborado tiene buena estabilidad microbiológica. Los valores encontrados en el estudio, resultaron menores a los establecidos para cereales, concluyendo que las barritas energético multicereal es un alimento que se debe incluir en la lonchera escolar, es inocuo, y de buen valor nutritivo.

Es responsabilidad, entonces de los padres de introducir este alimento en la ración alimentaria del niño, ya que por su buena aceptabilidad, $100 \mathrm{~g}$ de este producto que equivale a aproximadamente 2 barras, cubre aproximadamente el $30 \%$ y $25 \%$ de los requerimientos de proteínas y fibra alimentaria del preescolar y escolar. Lo ideal es que tanto preescolares como escolares lo consuman frecuentemente en la lonchera escolar, como sustituto de las galletas y pasteles.

\section{CONCLUSIONES}

No existen diferencias significativas en la aceptabilidad global de las barritas formuladas: "multibar-1", "multibar-2", "multibar-3". Los cambios sensoriales, estadísticamente son no significativos, por encontrarse la probabilidad dentro del área de aceptación del $5 \%$.

Las barritas energético-proteico mualticerel, presentaron un contenido de proteínas, de 9,65 $\mathrm{g} \%$ $\pm 0,257$ (Multibar-1), 11,42 g\% $\pm 0,326$ (Multibar-2) y $11,36 \pm 0,369$ (Multibar-3); grasas, de 9,18 g\% \pm 0,178 (Multibar-1), 10,83 g\% \pm 0,874 (Multibar-2) y 11,14 \pm 0,326 (Multibar-3), y fibra alimentaria de 7,58 $\mathrm{g} \% \pm$ 0,$215 ; 7,93 \mathrm{~g} \% \pm 0,262$ y $8,16 \mathrm{~g} \% \pm 0,226$, respectivamente.

La ingesta de $100 \mathrm{~g}$ de este producto que equivale a aproximadamente 2 barras, cubre el $30 \%$ y $25 \%$ de los requerimientos de proteínas y fibra alimentaria del preescolar y escolar. 


\section{REFERENCIAS BIBLIOGRÁFICAS}

AOAC (2004). Association of Official Agricultural Chemists. Official methods of analysis of the -AOAC. 15th ed. AOAC, Washington.

Asociación de Consumidores Lima- Metropolitana (2011). Galletas-Snack. Inf. Téc. Lima.

Borjas, G.M. (2012). Desarrollo de una barra de cereal con miel y polen destinada para el mercado infantil. Proyecto especial de graduación del programa de Ingeniería en Agroindustria Alimentaria, Escuela Agrícola Panamericana, Zamorano, Título de Ingeniera en Agroindustria Alimentari. Honduras. 24 p.

Calisto, L.A (2010). Desarrollo de producto snack a base de materias primas no convencionales poroto (Phaseolus vulgaris L.) y quinua (Chenopodium quinoa willd) memoria para optar al título de Ingeniero Universidad de Chile- Facultad de ciencias químicas y farmacéuticas. Laboratorio de procesos de alimentos. Santiago de Chile

Codex (2006). Norma de codex para alimentos elaborados a base de cereales para lactantes y niños pequeños codex stan 074 - 1981, Rev. 1 - 2006; 1(1):110

Collazos, C. et al (2009). La composición de alimentos de mayor consumo en el Perú. Instituto Nacional de Nutrición. Lima-Perú.

Coronil C. (2006). "Desarrollo de una Barra de Cereal tipo "snack" utilizando Quinua (Chenopodium quinoa Willd) y Nuez (Juglans regia) Variedad Semilla California. Estudio de Vida Útil y Determinación del Aporte Calórico/Protéico de la Formulación Optimizada". Universidad de Chile. Santiago. Chile.

FAO/OMS (2008). Necesidades de energía y de proteínas. Informe № 724 Roma - Italia.

Franco, F. M. (2002). Barritas de soja. Gualeguaychú. Universidad Nacional de Entre Ríos. 2002
Gutierrez, P. (2007). "Elaboración de galletas con semilla de chía (salvia hispánica) como alimento funcional con aporte de ácidos grasos omega-3"

memoria para optar al Título de ingeniero en alimentos . Universidad de Chile Facultad de Ciencias Químicas y Farmacéuticas Departamento de Ciencia de los Alimentos y Tecnología Química santiago-chile 2007.

CMSF. (2006) Ecología microbiana. Edit AcribiaZaragoza .España.

INE (2010). "Snacks Saludables". Noviembre/Diciembre. Pág. 40. CTG.Centro Tecnológico Granotec. Santiago. Chile.

INDECOPI (1985) Norma tecnica peruana 206.001 galletas-Requisitos .

INDECOPI. (1976) Norma tecnica peruana 205.044 harinas sucedáneas. procedentes de leguminosas de grano alimenticio.

Larrabure, G. "Publicidad y Obesidad Infantil" Rev. Vitonica alimentación, deporte y salud. Brasil. 2008.

López, L. y Suárez, L (2002). Galletas con valor nutricional agregado. Revista Ind. Data Perú. 2002; 5(1):3-7.

Román, T. (2009). Conocimientos y Razones de Compra de Barritas de Cereal en Adolescentes entre $12 y$ 18Años de la Ciudad de Concordia.

Román, M., y Valencia, F. (2006). Evaluación de galletas con fibra de cereales como alimento funcional. Rev. Vitae de la Facultad de Química Farmacéutica. Univ. De Antioquia. Colombia, 13(2):36-43.

ASPEC (2008). Cómo aprovechar la piel de frutas y verduras. Fundación Eroski Rev. Consumer International. España

ASPEC (2010) Asociación de Consumidores. Santiago de Chile. 